\title{
Observations with self-embedded sentences using written aids
}

\author{
ROY FREEDLE ${ }^{2}$ AND MARLYS CRAUN \\ EDUCATIONAL TESTING SER VICE
}

\begin{abstract}
Self-embedded sentences of any degree beyond one are permitted by the syntactic rules of English, yet previous studies report that Ss typically reject these sentences as being ungrammatical and, in addition, often cannot recover the meaning of these sentences. The present paper investigated the possibility of introducing specially structured self.embedded sentences as "aids" to the discovery of the structure of more complex self-embedded sentences. The group of $S s$ who received these aids performed significantly better in discovering the subject-verb and subject-object assignments in the complex sentences (many achieved perfect scores) in contrast to $S s$ who did not initially receive these aids / these latter $S$ s obtained close to the minimum possible scores).
\end{abstract}

Attention to psycholinguistic theory (Miller \& Chomsky, 1963; Fodor \& Garrett, 1967) has been drawn to the difficulty that Ss experience when they are asked to recall or to interpret the meaning of self-embedded (SE) sentences. The reason interest has focused on this difficulty is that SE sentences are known to be fully grammatical structures inasmuch as the rules of English grammar permit such structures to be generated. Nevertheless, considerable difficulty is experienced by language users when they are asked to paraphrase sentences that contain two self-embedded clauses (Fodor \& Garrett, 1967); with three self-embeddings, these sentences are totally rejected by language users as being ungrammatical (Blumenthal, 1966). Magnitude estimations of sentence grammaticality (Marks, 1968) also confirm this view that sentences with two or more self-embeddings are perceived as being ungrammatical constructions.

SE sentences are formed by placing one phrase totally within another phrase of the same type. For example, the following sentence exhibits self-embedding of degree one: "The doctor whom the nurse aided cured the disease." Rules of English grammar permit this embedding process to go on indefinitely so that one can end up, for example, with the following sentence that contains three SE clauses: "The doctor whom the nurse whom the woman whom the man addressed praised aided cured the disease." Such a sentence is extremely difficult to understand, if at all; typically, as already mentioned, experimental Ss reject such sentences as being ungrammatical (Blumenthal, 1966). A typical error that Blumenthal's Ss made was to make "nurse," "woman," and "man" modify "doctor"; another error involved perceiving "doctor," "nurse," "woman," and "man" as forming a compound subject and linking the four verbs together as a compound verb. Using the data of eight Ss, Blumenthal found that only $15 \%$ (24 out of 160 observations) of the two rewritten innermost clauses had assigned the verb to its correct subject. Clearly this finding deserves further study.

Miller and Chomsky (1963, p.471) observed that sentences of natural languages that contain self-embedding beyond a certain number should be impossible for the unaided native speaker to understand unless he is provided with certain aids. The nature of such "aids" has often been interpreted in terms of procedures that help overcome the limitation of short-term memory capacity by, for example, such devices as immediate recall (e.g., Menyuk, 1963); it is possible to consider the concept of linguistic "aids" in a somewhat broader sense as being any procedure or materials that overcome a performance problem and lead the language user to appreciate that his internalized rules of the language (competence) permit certain sentence constructions or sentence interpretations. In this broad sense, an example can be cited from Chomsky. In sentences such as "I had a book stolen," few language users "may be aware of the fact that their internalized grammar in fact provides at least three structural descriptions for this sentence. Nevertheless, this fact can be brought to consciousness by consideration of slight elaborations of [this] sentence... for example: (i) ... 'someone stole a book from my car'; (ii) ... 'I had someone steal a book'; (iii) 'I almost had a book stolen, but they caught me leaving the library with it,' that is, 'I had almost succeeded in stealing a book.' In bringing to consciousness the triple ambiguity of [this sentence] ... in this way, we present no new information to the hearer and teach him nothing new about his language but simply arrange matters in such a way that his linguistic intuition, previously obscured, becomes evident to him [Chomsky, 1965, pp. 21-22]."

In the light of these observations about linguistic "aids," one explanation of
Blumenthal's results is that his Ss were not provided with sufficient aids or clues to help them in understanding the triple SE sentences, which admittedly are unfamiliar structures in ordinary English usage.

The purpose of the present study was to investigate whether or not performance on rewriting triple-SE sentences could be greatly improved by prior presentation of sentences having only one or two self-embeddings.

The authors reasoned, for example, that if paired sentences of Types (a) and (b) below were used on consecutive trials early in testing, the relationships they bore to each other would provide a powerful clue towards the comprehension of SE sentences:

Type (a). "The woman that the boy complimented despised someone."

Type (b). "The policeman that the woman that the boy complimented despised arrested the drunk."

Notice that the italicized words from (a) appear in the same order in sentence (b).

Clearly, what we hoped to convey by this pairing of "prompting" sentences was the following. In sentence (a), "someone" can be replaced by the noun phrase of the matrix sentence ${ }^{3}$ in example (b), namely, "The policeman." Notice that the experimental $\mathbf{S}$ is left to discover this fact for himself; i.e., he is nowhere informed that "policeman" is the correct object of "The woman despised . .." in sentence (b). Also, by placing sentences (a) and (b) together, the $S$ is further aided because he can immediately convince himself that the underlined words and their ordering in (b) are perfectly acceptable and meaningful when transferred to a sentence of type (a). We assume, of course, that single $S E$ sentences like (a) are completely acceptable to adult native speakers of English (Marks, 1968). His results indicated that Ss do accept sentences with one SE clause as being fully grammatical. Hence, such sentence pairings should greatly aid the $S$ in discovering double-SE structures as exemplified by sentence type (b). Whether or not he will also transfer this presumed insight for the subsequent triple-SE sentences is one of the main motivations for the present study.

\section{Subjects}

\section{METHOD}

Nineteen Princeton male 
Table 1

List of Test Sentences.

1. The dentist whom the man aided extracted the tooth.

2. The pianist whom the boy admired played the sonata.

3. The woman whom the boy complimented despised someone.

4. The policeman whom the woman whom the boy complimented despised arrested the drunk.

5. The singer whom the seller cheated applauded someone.

6. The politician whom the singer whom the seller cheated applauded addressed the audience.

7. $b$ The businessman whom Fred whom Bill whom Pete trusts follows obeys warned the engineer.

8. The florist whom the watchmaker whom the jeweller whom the photographer assists trains entertains invited the electrician.

9. The visitor whom the soldier whom the girl whom the general sees impresses saluted the troops.

10. The playwright whom the editor whom the novelist whom the poet advises summons bothers feared the audience.

a. The first six sentences listed (in the order given) formed Part 1 of Form $A$ and formed Part 2 of Form $B$ (in the same order).

$b$. The last four sentences (in the order given) formed Part 2 of Form $A$ and formed Part 1 of Form $B$ (in the same order).

undergraduates ${ }^{4}$ were tested; their ages ranged from 18 to 21 years. Each $S$ was paid $\$ 2.00$ for participation in a $1-\mathrm{h}$ session.

\section{Materials}

Ten test sentences were constructed that varied in number of self-embeddings. Four sentences had one self-embedding, two had two self-embeddings, and the remaining four had three self-embeddings. These last four were slight modifications of sentences taken from Blumenthal's (1966) list of test sentences. Table 1 lists the test sentences. The modifications involved (1) changing the relative pronouns introducing each self-embedded clause from "that" to "whom," and (2) making all nouns singular so as to remove all cues regarding which subject and verb belonged together for each embedded clause. To give one example: "The florist that the watchmaker that the jeweller that the photographers assist trains entertains invited the electrician," was changed to read, "The florist whom the watchmaker whom the jeweller whom the photographer assists trains entertains invited the electrician." The main reason for substituting the relative "whom" for "that" stemmed from the results of a pilot study wherein some Ss questioned whether "that" is the appropriate relative. Since the concern of this study was the comprehension of SE sentences, it seemed desirable to remove any irrelevant and potentially distracting elements (such as a dubious choice of relative pronoun) and to remove any unintended cues regarding subject-verb agreement stemming from singularity or plurality of the clausal subjects-cues that occurred in a nonsystematic way among Blumenthal's sentences.

\section{Procedure}

Two orders of the 10 test sentences were formed: Form A, Part 1, consisted of the one- and two-SE sentences, while Part 2 consisted of just the triple-SE sentences. Form B, Part 1, consisted of just the triple-SE sentences, while Part 2 consisted of the one- and two-SE sentences.

The Ss were assigned randomly to the two orders based upon order of appearance for testing.

When each $S$ had completed Part 1 for either order, he handed this in to the $E$, and then he received Part 2 of the test.

\section{Instructions}

The main instructions were the same for both orders and for both parts within each order. They were as follows, "Each of the following sentences consists of two or more sentences which have been combined into a single sentence. Your task is to rewrite each of the following sentences without changing the meaning of the sentences. You can add punctuation and change or add words if necessary, but if you do, the essential meaning of the

Table 2

Number of Correct Subject-Verb and Subject-Object Assignments

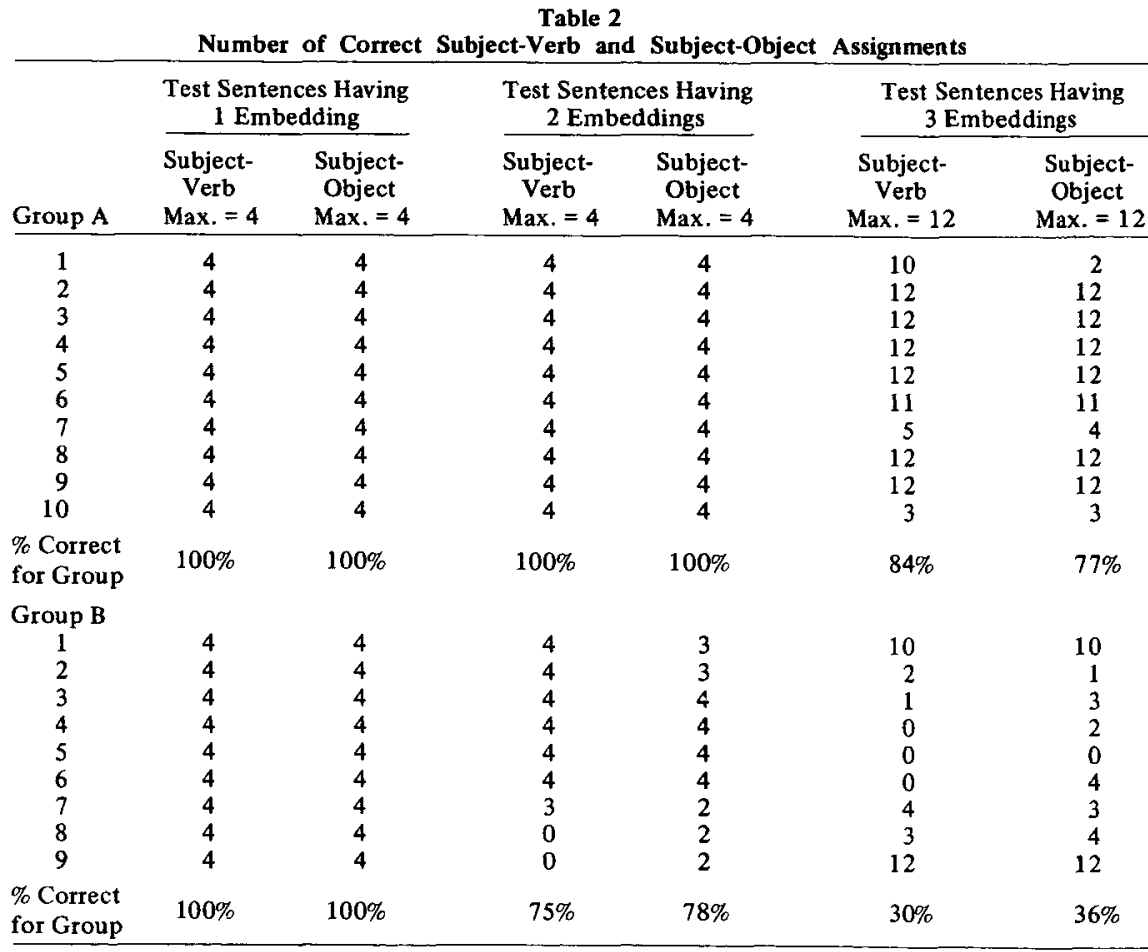

sentence must be the same as the original sentence. In rewriting you can use as your main criterion the following: Try to state the information in each sentence so that a listener may more easily comprehend the meaning." emphasized to the Ss that they must rewrite the sentences in the order listed; further, they were cautioned that simply adding punctuation to the test sentence did not constitute a rewriting.

\section{RESULTS}

The number of correct subject-verb and subject-object assignments in each $S$ 's rewritten sentences was found. Only the embedded clauses were scored, i.e., the matrix sentences were not included in the analyses.

Table 2 lists the number of correct subject-verb and subject-object assignments for the single-, double-, and triple-SE sentences for each of the two orders of presentation (Group A received Form A, Group B received Form B).

For the triple-SE sentences, a Fisher Exact Probability Test between the distribution of the sum of the correct subject-verb and subject-object pairings for each $S$ in Group A compared with the distribution of sums from Group B indicated that the median number correct for Group A was significantly higher than that for Group B ( $p<.025$, one-tailed).

A similar Fisher test of the distribution of sums of correct subject-verb and
Supplemental instructions given verbally 
subject-object assignments made by Group A in comparison with Group B for just the double-SE sentences indicated that the median of Group A was significantly higher than that of Group B $(p<.05$, two-tailed).

\section{DISCUSSION}

It is clear that including the single- and double-aid sentences prior to exposure to the triple-SE sentences leads to a significant improvement in the ability of Ss to comprehend the meaning of the sentences in contrast to the poor comprehension shown by Blumenthal's Ss and the Ss in Group B of the present study. Table 2 indicates that each $S$ obtained either close to the maximum possible score or the minimum possible score for both the subject-verb and subject-object pairings. Hence, it appears that the $S$ either fully knew or fully discovered the correct rule for rewriting SE sentences or he did not.

It is interesting to find that $S s$ in Group B performed significantly worse on their rewritings of the double-SE sentences when these followed the triple-SE sentences. Presumably, their difficulties in dealing with triple-SE sentences interfered with their ability to benefit from the specially structured paired aid sentences that followed.

Some interesting issues are raised when one considers the rapidity with which most of the Ss in Group A grasped the structural rule for SE constructions. On the one hand, since no knowledge of results was provided anywhere in the test, the Ss clearly had to infer the rule from the materials provided them and then had to generalize this rule to triple-SE sentences in order to achieve perfect (or close to perfect) comprehension scores as assessed by their subject-verb and subject-object pairings. It is intriguing to consider the possibility that the rule was discovered in all-or-none fashion by just a single presentation of the paired aid sentences. However, the fact that not all Group A Ss responded appropriately when triple-SE sentences were given (even though they correctly rewrote the aid sentences) suggests that these Ss had not yet discovered the general rule governing SE structure but, instead, had discovered a "rule" specific to the number of embeddings in the aid sentences.

\section{REFERENCES}

BLUMENTHAL, A. L. Observations with self-embedded sentences. Psychonomic Science, 1966, 6, 453-454.

CHOMSKY, N. Aspects of the theory of syntax. Cambridge: M.I.T. Press, 1965.

FODOR, J. A., \& GARRETT, M. Some syntactic determinants of sentential complexity. Perception \& Psychophysics, 1967, 2, 289-296.

MARKS, L. E. Scaling of grammaticalness of self-embedded English sentences. Journal of Verbal Learning \& Verbal Behavior, 1968, 7, 965-967.

MENYUK, P. A preliminary evaluation of grammatical capacity in children. Journal of Verbal Learning \& Verbal Behavior, 1963, 2 , 429-439.

MILLER, G. A., \& CHOMSKY, N. Finitary models of language users. In $R$. D. Luce, $R$. Bush, and E. Galanter (Eds.), Handbook of mathematical psychology. Vol. 2. New York: Wiley, 1963. Pp. 419-491.

\section{NOTES}

1. This research was supported in part by the National Institute of Child Health and Human Development under Research Grant 1 PO 1 HDO1762.

2. Address: Educational Testing Service, Room R123, Princeton, New Jersey 08540.

3 . In the sentence "The woman that the boy knew despised someone," the matrix sentence is the "outer" structure which reads "The woman ... despised someone."

4. The authors would like to thank Barbara Witten for conducting the main study.

(Accepted for publication July 3, 1969.) 